\title{
Barriers and Facilitators to Uptake of Prostate Cancer Screening in a Kenyan Rural Community
}

\author{
Ruth Gathoni Mbugua ${ }^{1}$, Simon Karanja², Sherry Oluchina ${ }^{3}$ \\ ${ }^{1}$ Mount Kenya University, College of Health Sciences, Community Health Department \\ 2Jomo Kenyatta University of Agriculture \& Technology, School of Public Health \\ ${ }^{3}$ Jomo Kenyatta University of Agriculture \& Technology, College of Health sciences, Department of \\ Nursing Education Leadership Management \& Research.
}

Correspondence to: Dr. Ruth Gathoni Mbugua, Mount Kenya University, rmbugua15@gmail.com

Received: 14 Oct 2020; Revised: 21 Feb 2021; Accepted: 08 Mar 2021; Available online: 18 Apr 2021

\begin{abstract}
Background: Prostate cancer (PC) is curable with early detection, yet it remains a major public health problem globally and a leading cause of mortality among men. The objective of the study was to explore the barriers and facilitators to the uptake of prostate cancer screening among men aged 40-69 years in a rural community in Kenya. Methods: We utilized an explorative qualitative design and purposive sampling to select participants. Six focus group discussions (FGDs) and seven in-depth interviews were conducted among 59 men aged 40-69 years and key informants in Kiambu County, Kenya. Data was collected using a semi-structured guide and content analysis was done. Results: The facilitators of screening included experience of symptoms, proximity and prominence of cancer, accessibility, and advocacy. The barriers to screening included lack of knowledge, fatalistic beliefs, low risk perception, stigma, and male dominance factors. Conclusion: This study provides
\end{abstract}

\section{Introduction}

Prostate cancer (PC) is curable with screening and early detection, yet it remains a major public health problem globally as it is among the leading causes of cancer-related mortality among men worldwide. African men suffer disproportionately from PC with higher mortality reported among men in Sub-Saharan Africa $(1,2)$. In Kenya, PC is ranked as the most prevalent cancer in males with 2864 new cases vital information for the development of interventions to enhance shared decision-making in regard to PC screening. Capacity building of clinicians, task shifting and provision of well-coordinated affordable culturally sensitive screening services should be explored. The concerted effort among policy makers and all health care workers to overcome the stated barriers to screening is highly recommended.

Keywords: Barriers, facilitators, prostate cancer, screening, men, Kenya

Ann Afr Surg. 2021; 18(3): 130-136

DOI: http://dx.doi.org/10.4314/aas.v18i3.2

Conflict of Interest: None

Funding: None

(C) 2021 Author. This work is licensed under the

Creative Commons Attribution 4.0 International License. 
Services Task Force recommendations (6). The screening guidelines in Kenya recommend individualized risk-based screening through shared decision-making between the client and clinician among men aged 40-69 years (3). The screening rates, however, remain abysmally low despite high intention to screen (7-10). Unfortunately, the number of men diagnosed with advanced aggressive $\mathrm{PC}$ is on the rise with an alarming increase in mortality attributed to low uptake of screening $(3,11)$. Despite equivocal evidence given on the effects of PC screening on mortality, risk-based screening aimed at early treatment initiation is vital (7).

The low uptake of PC screening among Kenyan men despite a considerably high level of awareness remains a great puzzle in public health that requires further investigation. There is a paucity of studies on barriers to PC screening in developing countries. The few studies carried out in Kenya are quantitative, hence they lack a deeper exploration of the factors influencing screening. The success of PC prevention and control programs requires an in-depth understanding of contextual factors influencing uptake of screening. The study therefore applied a qualitative approach to explore context-specific barriers and facilitators to PC screening among Kenyan men in a rural community.

\section{Methods}

\section{Design}

We used a descriptive exploratory qualitative study design.

\section{Study site}

The study was conducted in Gatundu-North and Kiambu Sub-counties in Kiambu County, which are located in the central region of Kenya. The subcounties were selected as they have linked health facilities which offer PC screening services. The population in the study area is demarcated into Community Units (CUs) for the purpose of implementation of community health strategy. All the 17 CUs were included in the study.

\section{Study population}

The study participants included 59 men aged 40-69 years, the eligible age for screening in Kenya (3). The key informants (KIs) included members of the County and Sub-county Health Management Committee including public health nurses and officers and community health strategy coordinators.

\section{Sampling technique}

The focus group discussion (FGD) participants were purposively selected which aimed at ensuring heterogeneity and representation of various socioeconomic and demographics characteristics. The selection of the KIs was facility-based and included the key people involved in the implementation of the Community Health Strategy in the sub-counties.

\section{Data collection tools}

A semi-structured guide based on the key themes of the study was used to conduct the interviews in the months of March-April 2019. The key themes of the study which included the barriers and facilitators of the uptake of screening were included in the guide.

\section{Internal validity and reliability}

Lincoln and Guba criteria were used for enhancing trustworthiness. Several debriefing sessions were held by the research team members, who used multiple coders ensuring consensus from the team members before the generation of themes. Referential adequacy was ensured by a review of the original data and findings by two members of the research team who had vast experience in qualitative data analysis and multiple reviews of the data by other research team members before generation of codes to ensure the credibility of the data. Operationalization was also done through member checking at the end of interviews and keeping an audit trail of all the steps undertaken during analysis to ensure rigor. Researcher and methodological triangulation was also done (12). 


\section{Data collection procedure}

We conducted six FGDs which consisted of 59 community members and seven individual in-depth interviews with KIs and achieved saturation with a total of 66 participants. Each FGD was made up of the principal investigator and two research assistants. The FGD was conducted in a private set-up within the linked health facilities at the study site. The FGD was made up of nine to eleven participants. The average duration was 82 minutes. The KI interviews lasted between 30 and 45 minutes. All the interviews were audio recorded and transcribed verbatim.

\section{Ethical approval}

The study was approved by the Jomo Kenyatta University of Agriculture and Technology Institutional Ethics Review Committee (JKU/2/4/896B), the National Council of Science and Technology (NACOSTI P/19/71673/28322) and the Ministry of Health. Participant's autonomy and privacy were maintained throughout the study and written consent was sought following the explanation of the purposes, benefits, risks, and ground rules of the session by the moderator.

\section{Data analysis}

The study applied inductive thematic analysis. The transcripts underwent de-identification of participants with codes. The research team immersed themselves in the data to enhance familiarization and triangulation of the data. The data was then coded independently by two researchers using the grounded theory (12). The final codes were generated based on consensus from the research team which included experienced researchers. The coding entailed the analysis of specific statements and their categorization into themes. This was followed by searching for the themes and researcher triangulation coupled with the diagrammatic representation of the connection of themes for further interrogation. Then a review of the themes and sub-themes was done and comparison with the raw data and the transcripts was done multiple times before the generation of codes. The main themes were then named and defined through consensus of the research team members and consultation of two experts in the subject. This was followed by the final analysis of the data using the established themes. The six steps of data analysis as guided by Braun and Clarke were applied in the study (12). The analysis also embedded some direct quotations of the participants to reflect their opinions.

\section{Results}

The socio-demographic characteristics of the respondents are presented in Table 1.

Table 1. FGD participants socio-demographics characteristics

\begin{tabular}{lll}
\hline VARIABLE & CATEGORY & TOTAL \\
\hline Age in years & $40-49$ & $25(42.4)$ \\
\hline & $50-59$ & $21(35.6)$ \\
\hline Marital status & Married & $48(82.0)$ \\
\hline & Single & $4(6.8)$ \\
\hline & Widowed & $3(5.0)$ \\
\hline Religion & Separated & $4(6.8)$ \\
\hline & Christian & $49(83.1)$ \\
\hline & Traditionalist & $4(6.8)$ \\
\hline Education & Muslim & $6(10.1)$ \\
\hline & None & $6(10.1)$ \\
\hline & Primary & $20(33.9)$ \\
\hline & Secondary & $27(45.8)$ \\
\hline
\end{tabular}

FGD, focus group discussion.

\section{Facilitators to uptake of PC screening}

Four themes emerged as facilitators of PC screening which included experience of symptoms, proximity and prominence of cancer, accessibility of screening services and community advocacy as presented in Table 2. 
Table 2. Facilitators to uptake of screening

\begin{tabular}{ll}
\hline THEMES & SUB-THEMES \\
\hline Accessibility of screening services & Provision of free screening \\
\hline & Inclusion of screening in National Health Insurance \\
\hline Experience of symptoms & Provision of screening services in the peripheral facilities \\
\hline Proximity and prominence of cancer & Inclusion in medical camps \\
\hline & Presence of symptoms \\
\hline Community advocacy on PC screening & Death of family member/friend/community member \\
\hline & Witnessing bad experiences of a relative/friend/community member \\
& affected by prostate cancer \\
\hline
\end{tabular}

Experience of symptoms: The respondents reported the experience of symptoms as a facilitator to the uptake of screening as stated, "I was visiting the toilet all the time to pass urine, so I decided to go to hospital to get checked: The doctor recommended that I get screened for prostate cancer."

Proximity and prominence of cancer: The experience of negative outcomes from PC among family members or friends and having a close person diagnosed with PC was reported by participants as a facilitator to the uptake of screening. A participant stated: "I lost my friend recently who was very close to me from prostate cancer, so I decided to take up screening."

Accessibility of screening services: The provision of free screening services which are available in peripheral health facilities and the inclusion of screening services in the National Health Insurance Fund was highlighted by the majority of the respondents as a facilitator of screening. A participant stated, "If the government provides these screening services for free, many men, including myself, might consider taking up the test."

Advocacy on prostate cancer screening: The creating of awareness through various channels of communication in the community was highlighted by the participants as a motivator to the uptake of PC screening. "I was informed about prostate cancer screening in church, and since I had also heard about it on the radio, I decided to go for the test."

\section{Barriers to uptake of PC screening}

Five themes emerged as barriers to the uptake of prostate cancer screening which included lack of knowledge, fatalistic beliefs, low perception of selfvulnerability, stigma, and male dominance factors as presented in Table 3.

\section{Lack of knowledge}

Lack of knowledge was reported by participants as a barrier to the uptake of screening. Myths and misconceptions also deterred the uptake of screening with the predominant cause of PC reported being the denial of conjugal rights as illustrated by one participant: "This disease is caused by lack of sex, so men don't want to go for screening because it will indicate their sexual life has a problem."

\section{Perception of fatalism towards PC}

Fatalistic beliefs were reported as a barrier to screening. The participants seemed to perceive a diagnosis of PC as a death sentence as expressed by a participant: "With cancer in the equation, the denominator is always death, I fear cancer more than any other disease." A key informant stated, "In the community people equate cancer to death, which has contributed to the majority of the men not undertaking screening and resulting in using herbal medication. 
Table 3. Barriers to uptake of PC screening

\begin{tabular}{ll}
\hline THEMES & SUB-THEMES \\
\hline Lack of knowledge and awareness & Lack of knowledge on PC disease \\
\hline & Lack of information on screening (where/methods/cost) \\
\hline & Confusion of PC with being an "old man's disease" (BPH) \\
\hline Perception of fatalism towards PC & Myths and misconceptions on etiology of prostate cancer (denial of conjugal rights) \\
\hline & Pessimism towards PC \\
\hline Low perception of self-vulnerability & Perception of death inevitability on diagnosis/death sentence \\
\hline & Fear of a diagnosis of PC \\
\hline Male dominance factors & Consider themselves at low risk \\
\hline & Lack of symptoms \\
\hline & Association of sickness with femininity \\
\hline Stigma & Masculinity threatened by low sexual performance \\
\hline & Secrecy among men \\
\hline & Preference of older males for screening \\
\hline & Social isolation due to shame \\
\hline
\end{tabular}

\section{Low perception of self-vulnerability}

Low perception of the risk of $\mathrm{PC}$ was reported as a barrier to the uptake of PC screening. One participant stated: "I have not been screened since I believe that I cannot get this disease after all, I take lots of healthy juices and my marriage is okay, you know what I mean..." A KI asserted, "The medical camps have seen a very low turn-out of men for cancer screening since the majority don't think they are at risk."

\section{Male dominance factors}

Male dominance factors emerged as barriers which included threatening of masculinity due to poor sexual performance, association of sickness with being female, and screening by older male clinicians. As reported by a participant: "There was a time there was a medical camp offering cancer screening in our village, and I did not see men going to be screened, most of these things are for women."

\section{Stigma associated with PC}

Stigma associated with PC was also cited as a barrier due to its association with sexual behavior. A participant stated: "Who really wants to go for screening? It will be like you are informing other people of your inability to perform sexually, it's too shameful for any man."

\section{Discussion}

Our study identified several barriers and facilitators to the uptake of PC screening in a rural community in Kenya. Experience of symptoms, proximity and prominence of cancer, accessibility of screening services, and advocacy were reported as facilitators of screening similar to those reported in other studies (13, 14). Clinicians should engage at-risk men presenting with urinary symptoms in shared decision-making in line with the screening guidelines (3). The provision of affordable screening services and the inclusion of peripheral facilities can be explored to circumvent the barriers to accessibility of the services. Public health awareness on PC should be incorporated in routine care and reinforced in the community through a collaboration of clinicians and community-based health workers.

In the study, lack of knowledge on PC was reported as a major barrier to uptake of screening. This finding is corroborated with studies conducted among men in the Sub-Saharan African region and Kenya (8, 9, 15). Participation of men in screening is highly dependent on their knowledge about PC and the benefits of early detection. Low perception of self-vulnerability to PC reported among this at-risk population has been reported in previous studies (16-18). Perception of self- 
vulnerability is critical in enhancing the uptake of screening (19). Targeted programs involving clinicians to reach at-risk men to enhance PC awareness and risk perception in the health care facilities and communities are urgently required.

Fatalistic beliefs reported as barriers to screening in our study have been reported previously $(9,20)$. Fatalism is mainly attributed to a lack of knowledge and negative outcomes of PC (21). Clinicians can overcome these fatalistic beliefs through education of at-risk men, timely diagnosis and navigation of patients which will contribute towards improvement in PC treatment outcomes $(20,22)$. The male dominance factors reported in the study have been documented in previous studies $(14,23,24)$. The preference of provision of screening services by older men was reported in the study. This can be explored by capacity building of male clinicians and inclusion of lower cadres like male nurses for provision of culturally acceptable screening services.

Clinician recommendation is fundamental in $\mathrm{PC}$ decision-making processes. Evidence shows that low PC screening is associated with weak physician recommendation (25). An important question given our results is the involvement of clinicians in awareness creation and recommendation of screening to at-risk men. A study conducted in Kenya reported that only $10 \%$ of the screened men had a recommendation from a health care provider while a majority were not involved in decision-making (7). The need for sensitization of all clinicians on the current screening guidelines cannot be over emphasized. The study recommends the development of decision aids to enhance informed decision-making among men and to facilitate the implementation of shared decision-making process among clinicians.

\section{Conclusion}

The study identified several barriers and facilitators to PC screening. Lack of knowledge remains a major barrier to screening as it contributes to myths and misconceptions, low perception of self-vulnerability, and negative beliefs. There is a need for targeted programs involving clinicians to reach at-risk men to enhance public awareness on PC. Integration of culturally sensitive education to regular care should be adopted. Capacity building of clinicians, task shifting, and provision of well-coordinated affordable screening services in peripheral facilities should be explored. The concerted effort among policy makers and all health care workers to overcome the stated barriers to screening is highly recommended.

\section{Strengths and limitations}

The strength of the study includes the use of multiple methods of data collection that enhanced the validity of the study. However, the selection of participants was purposive sampling. Nevertheless, the researchers ensured heterogeneity in regard to socio-economic and demographic characteristics and stratification using the CUs while the KIs were multi-disciplinary. The participants were from a rural community, and hence the results may not be entirely generalized to urban communities.

\section{References}

1. World Health Organization. WHO cancer mortality database (IARC). Geneva: World Health Organization. 2018.

2. Bray F, Ferlay J, Soerjomataram I, et al. Global Cancer Statistics 2018: GLOBOCAN estimates of incidence and mortality worldwide for 36 cancers in 185 countries. CanJ Clinic. 2018; 68(6): 394-424.

3. Ministry of Health Kenya. Kenya National Cancer Screening Guidelines. Nairobi: Ministry of Health. 2018.

4. Adeloye D, David RA, Aderemi AV, et al. An estimate of the incidence of prostate cancer in Africa: A systematic review and meta-analysis. PLoS One. 2016; 11(4): e0153496.

5. American Cancer Society. Cancer Facts and Figures 2018. www.cancer.org. 2018.

6. US Preventive Services Task Force, Grossman DC, Curry SJ, Owens DK, et al. Screening for Prostate Cancer: US Preventive Services Task Force Recommendation Statement. JAMA. 2018; 319(18): 1901-1913.

7. Mbugua RG, Oluchina S, Karanja S. Prostate cancer awareness and screening among men in a rural community in Kenya: A cross-sectional study. African J Urol. 2021; 27(1).

8. Wachira B, Meng'anyi LW, Mbugua RG. Knowledge, perception and uptake of prostate cancer screening: A cross sectional study at a level III Hospital in Kenya. Public Health Res. 2018; 8(4): 81-87. 
9. Mutua K, Pertet AM, Otieno C. Cultural factors associated with the intent to be screened for prostate cancer among adult men in a rural Kenyan community. BMC Public Health. 2017; 17(1).

10. Ministry of Health, Kenya. National Cancer Control Strategy 2017-2022. Nairobi, 2017.

11. Kenya National Bureau of Statistics, Ministry of Health/Kenya, National AIDS Control Council/Kenya, Kenya Medical Research Institute, National Council for Population and Development/Kenya, and ICF International. Kenya Demographic and Health Survey 2014. [Internet]. Rockville, MD, USA: 2015. Available from: http://dhsprogram.com/pubs/pdf/FR308/FR308.pdf.

12. Nowell LS, Norris JM, White DE, et al. Thematic analysis: striving to meet the trustworthiness criteria. Internat J Qual Meth. 2017; 16(1): 1-13.

13. Enaworu OU, Khutan R. Factors influencing Nigerian men's decision to undergo prostate specific antigen testing. African Health Sci. 2016; 16(2): 524.

14. James LJ, Wong G, Craig JC, et al. Men's perspectives of prostate cancer screening: A systematic review of qualitative studies. PLoS One. 2017; 12(11): e0188258.

15. Baratedi WM, Tshiamo WB, Mogobe KD, McFarland DM. Barriers to prostate cancer screening by men in SubSaharan Africa: An integrated review. J Nurs Scholarship. 2019; 52(1): 85-94.

16. Kinyao M, Kishoyian G. Attitude, perceived risk and intention to screen for prostate cancer by adult men in Kasikeu sub location, Makueni County, Kenya. Ann Med Health Sci Res. 2018; 8(3).

17. Khosravi V, Barati M, Moeini B, Mohammadi Y. Prostate cancer screening behaviors and its related beliefs among 50-to 70-year-old men in Hamadan: Appraisal threat and coping. J Educ Comm Health. 2018; 4(4): 20-31.

18. Muliira J, Al-Saidi H, Al-Yahyai A. Determinants of behavioral intentions to screen for prostate cancer in Omani men. Asia-Pacific J Oncol Nurs. 2017; 4(4): 348.

19. Koitsalu M, Eklund M, Adolfsson J, et al. Predictors of participation in risk-based prostate cancer screening. PLoS One. 2018; 13(7): e0200409.

20. Moreno PI, Yanez B, Schuetz SJ, et al. Cancer fatalism and adherence to national cancer screening guidelines: Results from the Hispanic Community Health Study/Study of Latinos (HCHS/SOL). Cancer Epidemiol. 2019; 60: 3945.

21. Kobayashi LC, Smith SG. Cancer fatalism, literacy, and cancer information seeking in the American public. Health Educ Behavior. 2015; 43(4): 461-70.

22. Tayel AA, El Sherbini HH, Abou Shosha AA, et al. The effect of applying preventive intervention based on Champion Health Believe Model on breast cancer fatalism, knowledge and screening behaviors among female employees. Am J Nurs Res. 2019; 7(5): 759-770.
23. Fish JA, Prichard I, Ettridge K, et al. Psychosocial factors that influence men's help-seeking for cancer symptoms: a systematic synthesis of mixed methods research. PsychoOncol. 2015; 24(10): 1222-1232.

24. Friedman DB, Thomas TL, Owens OL, et al. It takes two to talk about prostate cancer. Am J Men's Health. 2012; 6(6): 472-484.

Lee DJ, Consedine NS, Spencer BA. Barriers and facilitators to digital rectal examination screening among African-American and African-Caribbean men. Urology. 2011; 77(4): 891-898. 